\title{
LA MOVILIDAD URBANA EN LA CIUDAD DE LIMA La capacidad ambiental de la movilidad desde la forma de la ciudad
}

\author{
Zulema Conto Quispe \\ Universitat Politècnica de València \\ Fernando Gaja i Díaz; Rocío Cacho Cruz \\ zuconqui@doctor.upv.es
}

\section{RESUMEN}

Desde este artículo se abordan las diferencias significativas entre movilidad urbana motorizada y el medio ambiente en el que se desarrolla. El objetivo de la investigación es establecer para el área metropolitana de Lima; si la actual forma de la ciudad, desde los factores que determinan la movilidad, se asocian con costes ambientales y sociales específicos; y si estos repercuten en el desborde su capacidad ambiental. El diseño metodológico es de carácter exploratorio y descriptivo, a partir del marco teórico que explica el comportamiento de la movilidad en el área de estudio apoyada en una fuente de datos cualitativos y cualitativos secundarios.

Palabras clave: movilidad urbana, forma de la ciudad, capacidad ambiental.

\begin{abstract}
A partir de este artículo se abordan las diferencias entre la movilidad urbana motorizada y el entorno en el que se desarrolla. El objetivo de la investigación es establecer para el área metropolitana de Lima; si la forma real de la ciudad, a partir de los factores que determinan la movilidad, está asociada a costos ambientales y sociales específicos; y si estos tienen un impacto en su capacidad ambiental. El diseño metodológico es exploratorio y descriptivo, del marco teórico que explica el comportamiento de la movilidad en el área de estudio respaldada por una fuente de datos secundarios cualitativos y cualitativos.
\end{abstract}

Key words: urban mobility, city form, environmental capacity. 


\section{INTRODUCCION}

En los últimos años se ha empezado a cuestionar la visión convencional que se tiene del transporte, como un bien económico que solo busca incrementarse indefinidamente. Esta preocupación por el deterioro del medio ambiente a causa del tráfico rodado fue manifestada inicialmente por Colin Buchanan en 1961, quien introdujo el concepto de "capacidad ambiental" para referirse a la capacidad que tienen los diferentes tejidos en una ciudad para asimilar el tráfico rodado. Años después Estevan \& Sanz (1996), hablan de la contradicción esencial entre el transporte y la naturaleza, de donde surge el concepto de "capacidad de carga" como expresión de los limites ambientales de los ecosistemas naturales en relación con el transporte. Ambos términos "capacidad ambiental" y "capacidad de carga" se refieren a lo mismo.

Partiendo de estos estudios y de la revisión teórica sobre las variables que determinan la movilidad en las ciudades, se explica los costes ambientales y sociales, que se están generando en el área metropolitana de Lima (AML).

La hipótesis de partida sugiere que los mayores costes ambientales y sociales de la movilidad urbana en el AML; se atribuyen a la forma y configuración socio espacial de la ciudad, el cual está estrechamente ligado a procesos de expansión y dinámicas urbanas descontroladas asociadas a la ausencia o debilidad del Estado; situación que está llevando a superar su capacidad ambiental.

El objetivo de esta investigación es establecer para el AML, si la actual forma de la ciudad desde los factores que determinan la movilidad, se asocian con costos ambientales y sociales específicos; y si estos repercuten en el desborde su capacidad ambiental.

\section{MARCO CONCEPTUAL}

En las últimas décadas muchas investigaciones han evidenciado la estrecha relación entre el crecimiento de las ciudades y el desarrollo de la movilidad o transporte (ciudad - transporte); estas investigaciones han relacionado el incremento de la movilidad con variables como la densidad (variable la más analizada), se ha demostrados que existe una fuerte correlación entre la densidad y la distancia recorrida en vehículo motorizados. La diversidad o la mezcla funcional del suelo que promueve desplazamientos no motorizados, Cervero (1988). El diseño urbano que influye en la elección de los modos de desplazamiento, Cervero y Radisch (1996). Estas tres variables densidad, diversidad y diseño; son conocidas como los factores del entorno construido que más influyen sobre la demanda de los viajes; y fueron denominadas como las "3Ds" por Cervero y Kockelman (1997).

Esta lista posteriormente ampliada por Ewing (2007), quien menciona que se ha aportado abundante evidencia empírica que demuestran la existencia de otros factores como "accesibilidad del destino" y la "distancia al transporte público"; ampliándose la lista a las denominadas "5Ds". Sin cuestionar esta relación, algunos investigadores han demostrado que además de estos factores existen otros aspectos que son determinantes a la hora de explicar el comportamiento de la movilidad en las ciudades; como son las condiciones socioeconómicas Pouyanne (2006), y el sistema de transporte.

Finalmente Polzin (2004) realiza una revisión de la teoría y literatura desarrollada y elabora un modelo conceptual donde clasifica los factores en tres áreas principales: factores del uso del suelo, factores económicos sociales, y los factores del sistema de transporte (grafico 1). A partir de estos factores se va explicar para el área de análisis, si estos generan costes ambientales y sociales específicos. 


\section{Indirect Drivers of Travel Behavior}

Changes and VMT Growth

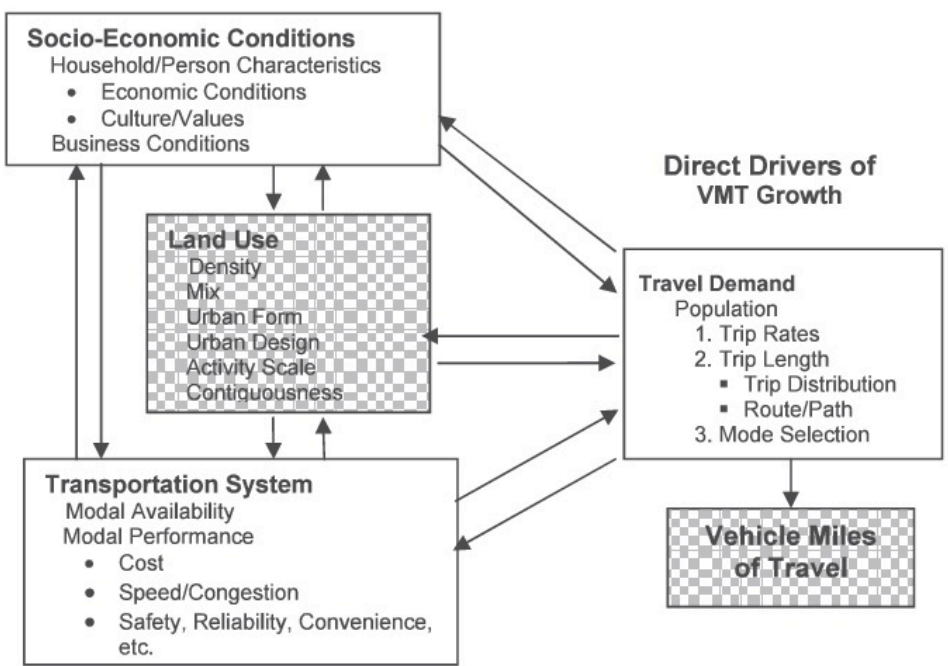

Grafico 1. Modelo conceptual de Polzin "factores que influyen en la demanda de la movilidad"“ Fuente: Polzin (2004)

\section{AREA DE ESTUDIO Y METODOLOGIA}

El área de estudio de esta investigación se ubica en el área metropolitana de Lima, cuenta con una población de $8 ` 894,412$ habitantes que representa el $30 \%$ de la población peruana, cifra que la convierte en la ciudad más poblada del Perú.

Es la quinta ciudad más poblada de América Latina, se localiza en la costa central del Perú, a orillas del océano Pacifico. Conformada por 49 distritos agrupados en cinco sectores, Lima centro, Lima norte, Lima este, Lima Sur, y el Callao.

\subsection{Metodología}

El diseño metodológico de la investigación es de carácter exploratorio y descriptivo; a partir del marco teórico se explicar el comportamiento de la movilidad y sus respectivos costes. Para ello se hace uso de una fuente de datos cuantitativos y cualitativos secundarios.

La hipótesis de partida sugiere que los mayores costes ambientales y sociales de la movilidad urbana en el AML; se atribuyen a la forma y configuración socio espacial de la ciudad, el cual está estrechamente ligado a procesos de expansión y dinámicas urbanas descontroladas asociadas a la ausencia o debilidad del Estado; situación que está llevando a superar su capacidad ambiental. Por lo tanto los costes ambientes y sociales tienen el papel de variable dependiente, mientras que la configuración de la forma urbana (factores que determinan la movilidad en la ciudad) representa las variables independientes (grafico 2).

\begin{tabular}{|c|c|c|}
\hline Variable independiente & \multicolumn{2}{|c|}{ Variable dependiente } \\
\hline Densidad & \multirow{6}{*}{$\begin{array}{l}>\quad \text { Costes ambientales } \\
>\quad \text { Costes sociales }\end{array}$} & \multirow{6}{*}{ Capacidad ambiental } \\
\hline Mezcla funcional & & \\
\hline Diseño & & \\
\hline Accesibilidad al destino & & \\
\hline Condiciones socioeconómicas & & \\
\hline Aspectos relacionados transporte & & \\
\hline
\end{tabular}

Grafico 2. Limites ambientales y sociales de la movilidad urbana desde la forma de la ciudad

Fuente: elaboración propia 


\section{LOS COSTES DE LA MOVILIDAD URBANA A TRAVES DE LA FORMA DE LA CIUDAD}

\subsection{Densidad}

Según el informe del Centro de Estudios Urbanos Estadounidenses sobre "Demografía de las áreas urbanas del mundo" para el año 2016, el área metropolitana de Lima se ubica en el puesto 29 con una población estimada de 10,950 habitantes; las ciudades latinoamericanas que la anteceden son Sao Paulo (20,605,00 hab.); Ciudad de México (20,230,00 hab.), Bueno Aires (14,280,000 hab.) y Rio de Janeiro (11,815,000 hab.); lista encabeza por Tokio con 37,750,000 habitantes. En cuanto a la densidad poblacional Daca encabeza la lista como la ciudad más densa del mundo seguida principalmente por ciudades asiáticas; Lima se encuentra en el puesto 172 con una densidad urbana de 11,900 hab/km² cabe mencionar que las densidades de las ciudades latinoamericanas son superiores a las grandes ciudades europeas e inferiores a las de grandes ciudades de países del Asia y África.

La densidad es uno de los factores del uso del suelo más fácil del entorno construido a ser medido, y por ello ampliamente utilizada. La densidad (bruta) de población en el área Metropolitana de Lima según estimaciones y proyecciones de población del Instituto Nacional de Estadística e Informática (INEI) al 2016 ${ }^{1}$ es de 3,379.5 hab/ $\mathrm{km}^{2}$; en sus distritos la población se encuentra distribuida de manera asimétrica en el tejido urbano, presentando áreas con densidad poblacional muy bajas que van desde $62 \mathrm{hab} / \mathrm{km}^{2}$ hasta otras con más de 26,117 hab/km²; el distrito con mayor densidad es Surquillo, seguido por Mi Perú y Breña, Santa Anita; mientras que los distritos de menor densidad son Punta Negra y Punta Hermosa pertenecen al sector de Lima sur.

\section{Costes ambientales - consumo del suelo}

Al hacer una revisión de la evolución histórica del crecimiento de la ciudad (población y superficie), se puede ver que durante la colonia y los dos primeros siglos de república se mantuvo un lento crecimiento, sin embargo en el siglo XX se da una explosión demográfica, así 1940 se constituye como un hito importante pues es a partir de ese año que la tasa de crecimiento se torna sostenida. En el grafico 3 se aprecia el crecimiento de población, superficie y área urbanizada; para 1940 se tenía una población de 661,508 habitantes; en 1961 aumento a 1,901,927; en 1993 llego a 6,434,323; y el censo nacional del 2007 arrojo la cifra de 8,556,109 habitantes; y según estimación del INEI para julio del 2010 fue de 9,160,384 habitantes.; así Matos Mar (2012) explica que entre 1940 y 1961, el área ocupada creció un 111.2\% con respecto a la 1931, debido a lo que los arquitectos y urbanistas denominan un ciclo de expansión vacía, que consistía en la incorporación de grandes extensiones de terreno agrícola al uso urbano, sin ocasionar una inmediata urbanización. En los últimos años a pesar que la tasa de crecimiento poblacional ha ido aumentando en menor porcentaje que la segunda mitad del siglo $X X$, se refleja una disminución en su expansión urbana, debido al proceso de consolidación y la ocupación del territorio en la conurbación de las zonas periféricas, así como por la densificación de sus actividades urbanas, además de la actividad residencial.

\footnotetext{
${ }^{1}$ El último censo de población desarrollado por el INEI corresponde al año 2017, cuyos resultados a la fecha no se encuentran disponible; se toman las estimaciones y proyecciones de población del INEI al 2016.
} 


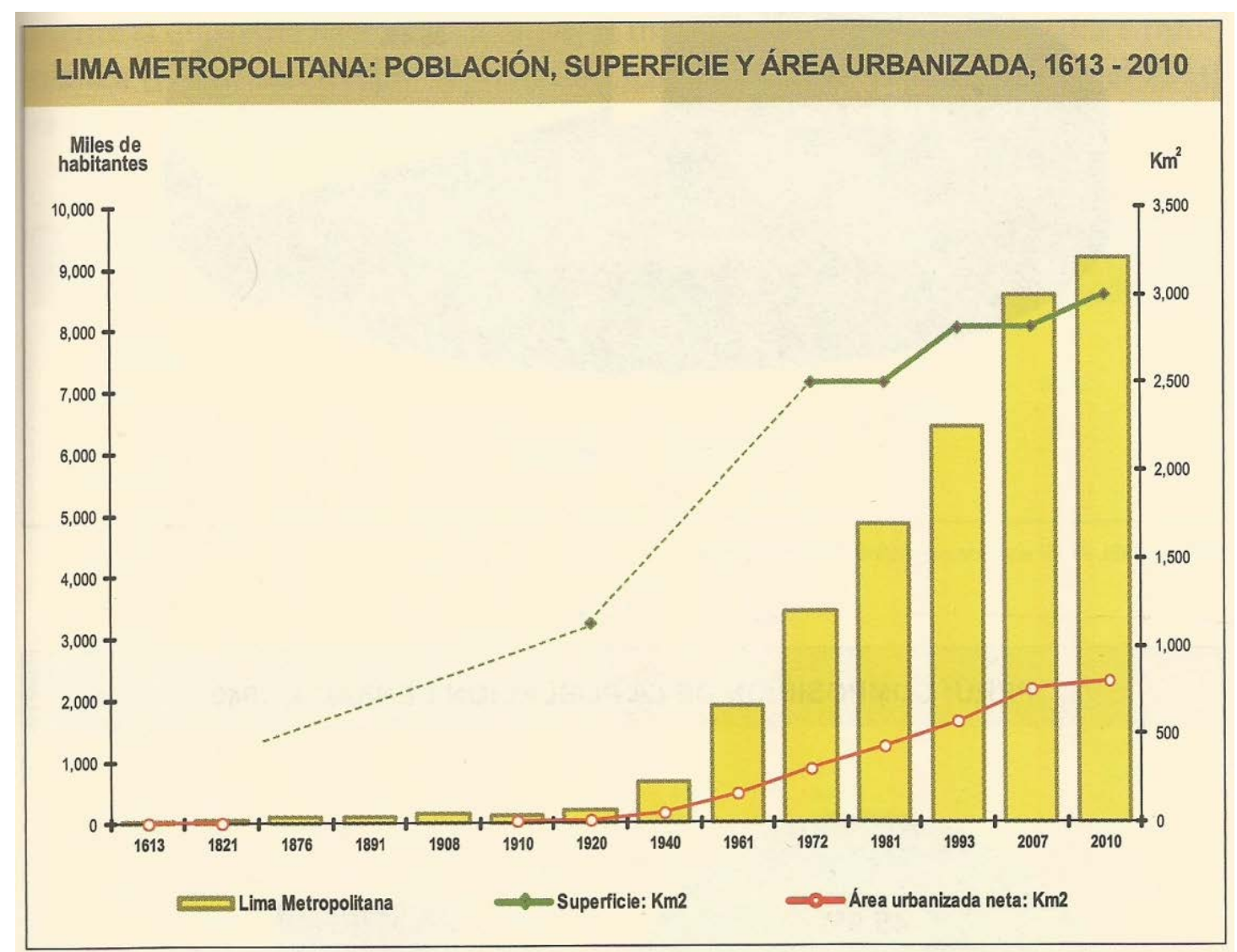

Grafico 3. Evolución de la población, superficie y área urbanizada de Lima Metropolitana Fuente: Matos Mar 2010

Esta explosión demográfica dio lugar a una expansión urbana basada en el crecimiento periférico de baja densidad; incentivando un excesivo consumo de suelo, especialización espacial, separación de usos del suelo, y la fragmentación del territorio. El alto consumo del suelo genera una creciente artificalización del espacio a través de la perdida de tierras agrícolas, bosques, zonas verdes, etc. situación que lleva a perdida de suelo libre; donde la construcción de viviendas, carreteras, etc. ocasiona el sellado del suelo, pudiendo tener gran impacto en los suelos colindantes modificando el curso del agua y contribuyendo a agravar la fragmentación de la biodiversidad, Garbiñe (2007). Así el área metropolitana de Lima entre los años 1940 al 1990 ha incrementado su superficie artificializada, en gran medida a través del consumo de suelo agrícola (sector de Lima centro), suelo desértico o con patrimonio arqueológicos (sectores de Lima norte, Lima sur y Lima este), el consumo del suelo, recurso ilimitado y difícilmente renovable, cuya conservación como suelo no construido supone preservar el territorio y aumentar la libertad de acción de las generaciones futuras, situación que debería ser de prioridad.

\section{Costes sociales - consumo de tiempo}

El desarrollo urbano de baja densidad tiene una estrecha relación con el incremento de la superficie; a mayor extensión se establece un alejamiento entre determinados usos del suelo y funciones urbanas, y por tanto mayores distancias a recorrer. El concepto distancia íntimamente relacionado al de accesibilidad resulta algo complejo de abordar; por un lado podemos hablar de distancia en el plano euclidiano, pero cuando se entra en una concepción relativa y hacemos referencia a un lugar distante, también puede hablar en unidades de tiempo.

Según el Banco de Desarrollo de América Latina, un latinoamericano promedio dedica aproximadamente 1 hora y 30 minutos al día para desplazarse de su casa al trabajo; también indica que en ciudades como Lima, Ciudad de México, Ciudad de Panamá, Bogotá y entre otras el $25 \%$ de los trabajadores invierte más de dos horas al día, en total, en trasladarse entre su casa y el trabajo. Como cualquier promedio estas cifras pueden esconder variaciones importantes; por ello al verificar los tiempos de desplazamiento según distrito se constata que aquellos con densidades más bajas tienen mayores tiempos de desplazamientos (grafico 4), estando aproximadamente entre 20 y 30 minutos el promedio de viaje al área central, mientras que toma entre 45 y 50 minutes desde los distritos periféricos; cabe resaltar que estos corresponden a la población de 
menor nivel socioeconómico, mientras que los distritos de las zonas más céntricas tiene un nivel socioeconómico más elevado; situación que evidencia que los estratos más pobres tiene más lejos todo y por tanto tienen que hacer más desplazamientos, mayor inversión de tiempo y mayor gasto de dinero. Así mientras los hogares con más ingresos gastan alrededor del 8,7\% de sus ingresos en transporte, los más pobres gastan el 42,7\% según Agencia de Cooperación Internacional de Japón (JICA).
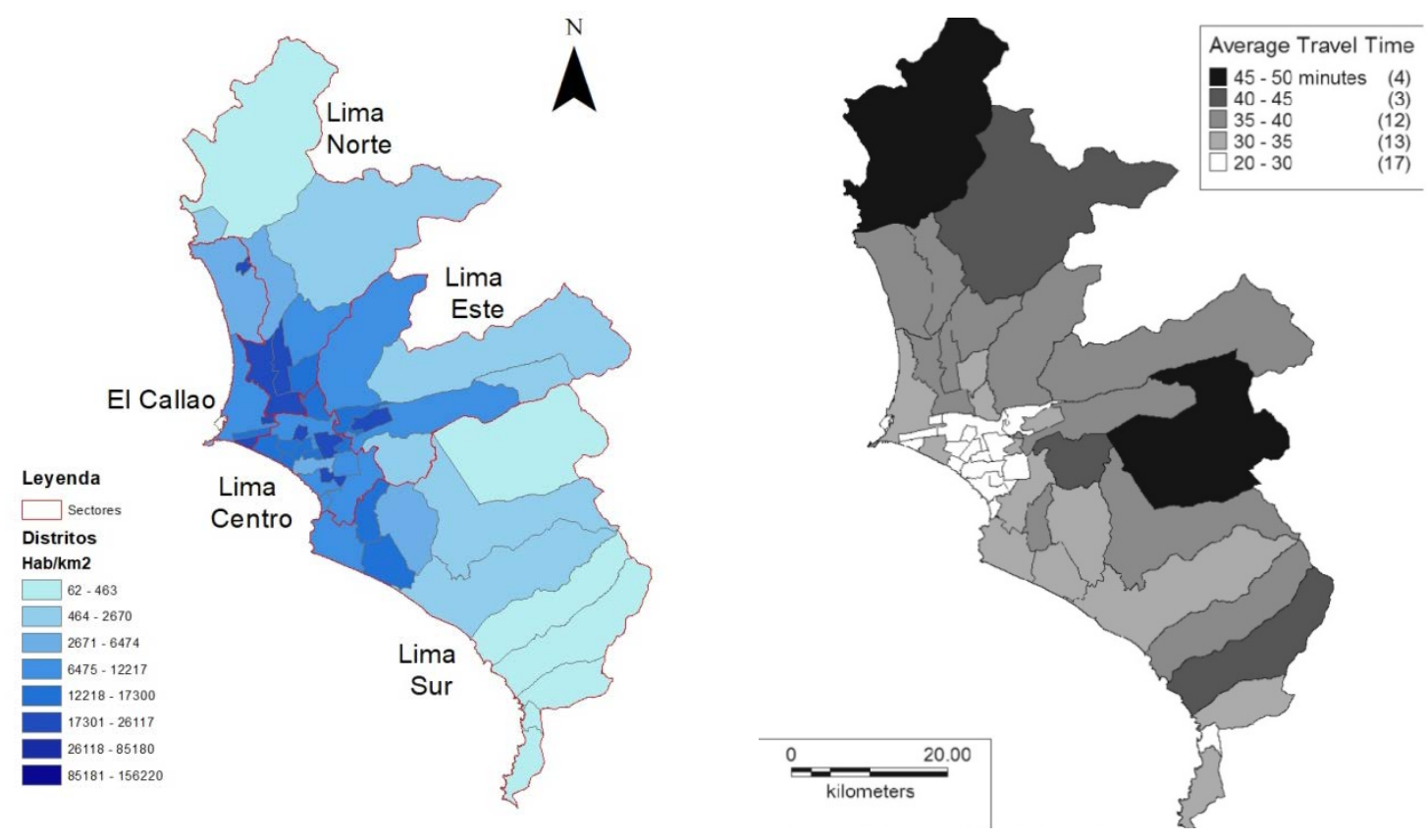

Grafico 4. Comparación de densidad de población y promedio de viaje por distrito del área metropolitana de Lima Fuente: Elaboración propia en base a INEI-2016, y JICA-2012

\subsection{Diversidad o mezcla funcional}

Desde su fundación la organización espacial de Lima estuvo basada en un solo centro, durante siglos el Centro Histórico de Lima cumplió funciones múltiples (sede de gobierno, administrativo, financiero, cultural, etc); a partir de la explosión demográfica de 1940 se llega a configurar una estructura radial metropolitana articulada por el Centro Histórico; el cual estaba rodeado por dos anillos concéntricos: un anillo interno definido por la antigua área urbana consolidada y un anillo externo definido por los distritos periféricos.

El área urbana consolidada, surge a partir del Centro Histórico que luego de muchas décadas como único centro urbano, en los años '80 entra en un proceso de deterioro de su configuración física y económica; y conforme se iba extendiendo la metrópoli, de manera tenue también los hacían las actividades urbanas. Así para los años '90 el patrón espacial se caracteriza por el crecimiento de múltiples centros especializados entre los que destacan, el distrito financiero en San Isidro donde se desarrollan las actividades informacionales que responde a las necesidades de una elite ejecutiva profesional; y el distrito de confecciones de Gamarra donde se desarrollan ciertas actividades industriales, que surge producto de la habilidad de empresarios y organizaciones locales para establecer reglas informales de cooperación (Chion, 2002). Conformando así un nuevo centro espacial que profundiza el carácter centralista de la metrópoli; en esta área urbana consolidada se encuentran el puerto del Callao, el aeropuerto internacional, y los distritos de estratos socioeconómicos altos y medios altos. Por otro lado el anillo externo caracterizado mayoritariamente por ser de baja densidad, surge de la migración interna entre los años 1940-1990, cuyo principal objetivo de urbanización fue la vivienda; estos sector inicialmente estuvieron infradotados de servicios básicos y carentes de equipamientos colectivos, y a medida que se fueron consolidando han ido adquiriendo cada vez más funciones urbana; están integrados en su mayoría por los distritos que conforman los sectores de Lima norte, Lima este, Lima sur, el Callao. 


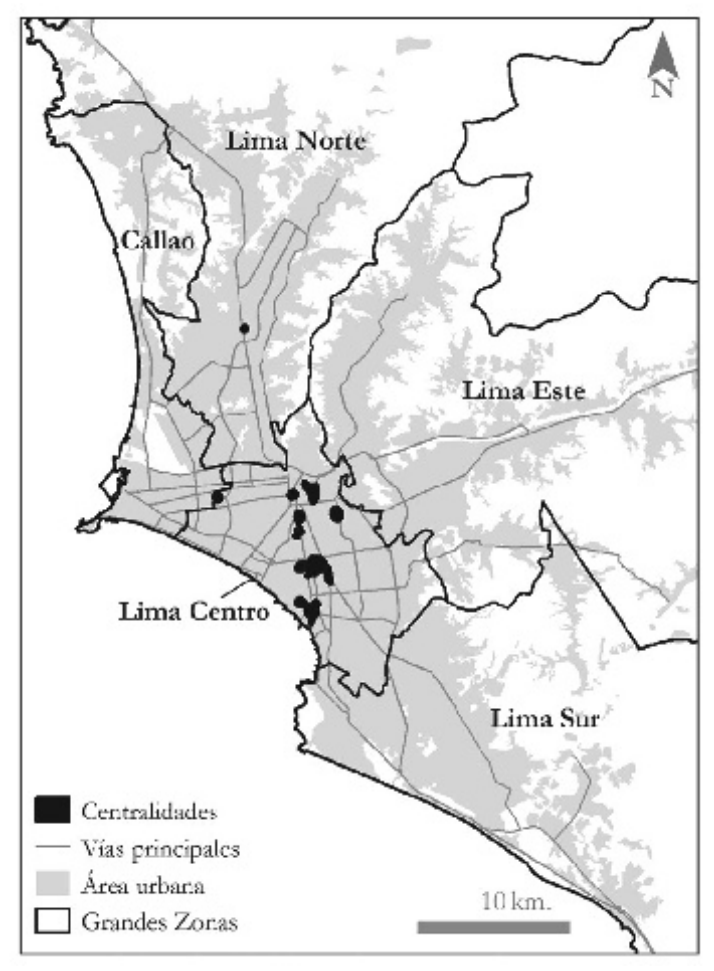

Grafico 5. Ubicación de principales centros según las grandes zonas de lima metropolitana Fuente: Vega Centeno (2015)

En los últimos años muchos investigadores han destacado el desarrollo de las nuevas centralidades en la metrópoli de Lima, de la organización en torno a un solo centro se ha pasado a formar una ciudad policentrica centralizada, así Gonzales y Del Pozo (2012) identifican grandes concentraciones del empleo (con funciones distintas pero complementarias) que se sitúan en la zona central de la metrópoli; los que se concentrados en una franja relativamente reducida. Por su parte Vega-Centeno (2017) habla de nuevas centralidades en el sector de Lima norte que le ha otorgado mayor dinamismo económico a este sector, sin embargo a pesar de su importancia como destino laboral o de abastecimiento, observa que aún desempeñan un rol secundario dentro de la estructura metropolitana.

De lo anterior cabe mencionar que los mayores centros metropolitanos se encuentran en su totalidad en el área central, lo que indica que pese a los cambios del siglo XXI Lima mantiene aún una estructura centralizada. Según Fernández \& Vilela (2015), el área central concentra la mayoría de las actividades urbanas de jerarquía metropolitana mientras que las de jerarquía local y zonal se ubican en el resto de zonas.

\section{Coste ambiental - fomento de modos motorizados}

Diversos estudios han demostraron que la diversidad de usos del suelo podría ser una herramienta útil para controlar los desplazamientos; así Cervero (1991) mencionaba que los altos niveles automovilísticos están asociados con los entornos construidos que tienen bajas densidades, usos individuales del suelo, y las altas prestaciones de estacionamiento; mientras que por el contrario los viajes a pie eran más comunes a los entornos con densidades altas, múltiples usos de la tierra, y aparcamiento limitado. Partiendo de este argumento para la metrópoli de Lima que a pesar de los débiles procesos de desconcentración de actividades mantiene una configuración centralizada; así el "centro" actúa como un imán de actividades lo que produce la necesidad de desplazamiento.

JICA para el año 2004 estimó 16,5 millones de viajes por día de los cuales el 75\% son motorizados; a nivel de sectores los porcentajes también son elevados, en Lima centro es de 83.55\%; Lima norte de 69,10\%; Lima sur de 67,97\%; Lima este de 68.51\% y para el Callao de 71.15\%. situacion que en el años 2012 se repite el número de viajes se ha incrementado a 22,5 millones de viaje por dia; y el principal destino sigue siendo el sector de lima centro. 
De los datos anteriores se aprecia que la falta de servicios y oportunidades en los demás sectores obligada a la población a desplazarse fuera de su zona de residencia realizando grandes desplazamientos en medios motorizados. Este hecho también fue evidenciado por Avellaneda (2009) quien a partir de un estudio en un asentamiento popular en el sector de Lima este; menciona que los comportamientos modales de las personas en situación de pobreza está estrechamente vinculados a la organización funcional del territorio urbano y a sus posibilidades económicas, lo que hace que desarrolle buena parte de su vida cotidiana lejos de su lugar de residencia.

\section{Coste social - segregación social}

En suma la estructura centralizada de Lima genera dos escenarios un área central y una periférica, así la separación de usos del suelo y las diferencias socioeconómicas (mayoritariamente estratos bajo y medio bajo) establecen ciertas limitaciones a los residentes en estas zonas, que se manifiestan en la aparición de fenómenos como a segregación y la exclusión social.

\subsection{Diseño}

La tercera "D" el diseño y su relación con la movilidad es algo ambigua en relación a las dos anteriores; mucho depende de la escala de análisis las variables a utilizar, para este ítem para va utilizar la "densidad de red de calles" al ser una variable que permite realizar un análisis a nivel metropolitano. Sobre la "densidad de red de calles" (census block density) Frank, Stone, \& Bachman (2000) mencionan que a mayor densidad de red de calles se producen menos viajes vehiculares; mencionan además que a medida que la red de calles aumenta en densidad los polígonos de bloques disminuyen en tamaño; así aquel patrón con pequeños bloques o la cuadricula tradicional produce menos viajes vehiculares puesto que reduce la distancia de ruta para todos los modos de desplazamiento al aumentar el número de opciones de ruta. Cabe acotar que el interés de los impactos de la red de transporte en los viajes son recientes, y los estudios son mucho menos numerosos que los estudios sobre los impactos del uso de la tierra (Ewing \&Cervero, 2000).

La urbe de Lima se encuentra ubicada en el centro de la zona costera del Perú, constituye el punto focal de una red de comunicaciones por carreteras conectando algunas ciudades costeras de norte a sur, y el centro del ámbito territorial del Perú (grafico 6). La actual red vial urbana de la metrópoli de Lima la define y regula el Ministerio de Transporte y Comunicaciones, y en el caso de la provincia de Lima la Ordenanza 341 del 2001, clasifica las calles. La clasificación de las vías es de carácter funcional, es decir se basa en la función que cumple cada una de ellas dentro de la estructura urbana, no se menciona su funcionalidad respecto al tránsito peatonal y condiciones ambientales, la definición que se hace de ella está orientada a sus posibilidades como medios de transporte. Así según la clasificación se tiene las vías expresas, arteriales, colectoras y locales.

\footnotetext{
${ }^{2}$ La densidad de red de calles, es la medida del número de bloques por milla cuadrada, los bloques generalmente se designan como los polígonos totalmente cerrados o delimitados por características cartográficas lineales, como las carreteras (vías).
} 


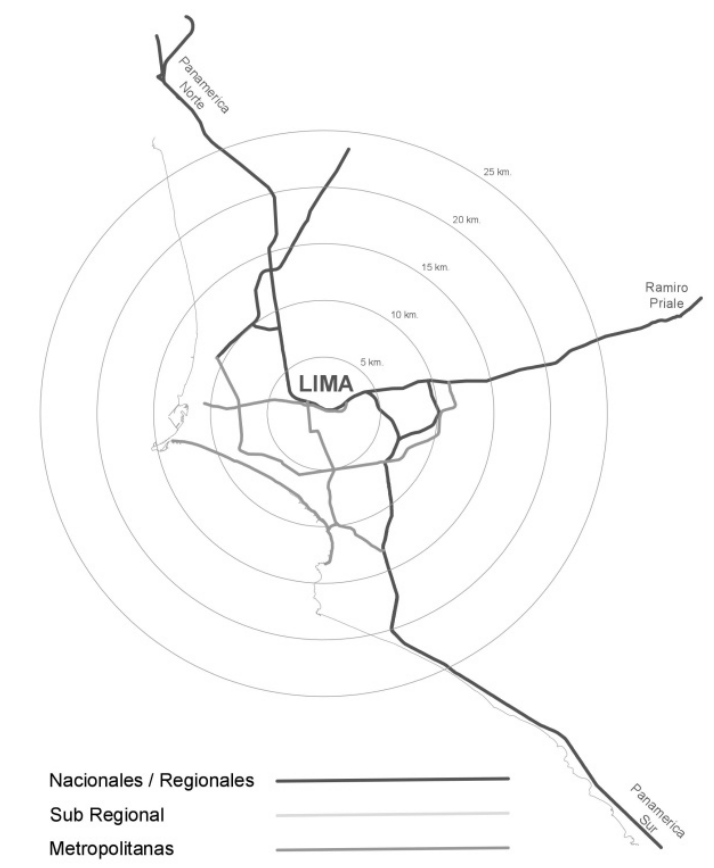

Grafico 6. Ejes viales primarios del área metropolitana de Lima

\section{Costes sociales}

\section{Segregación social}

Los países en vías de desarrollo se caracterizan por poseer redes de infraestructuras frágiles y desequilibradas; para el caso concreto de la metrópoli de Lima estas además de frágiles y desequilibradas son altamente excluyente.

Frágil; si bien la metrópoli de Lima está cubierta en su totalidad por algún tipo de red vial; las zonas periféricas están conectadas por vías de segundo y tercer orden; esto por el proceso de desarrollo urbano al que corresponden (generación espontánea por invasión) y que en la mayoría de los casos se dios sin control alguno. Otro aspecto es que no cumplen con la función para la que fueron proyectadas como es el caso de la Av. Javier Prado que tiene la calificación de vía metropolitana (red primaria) sin embargo actúa como colectora (red secundaria) puesto que las obras de implementación se encuentra inconclusas, en situación similar se encuentra la Av. Canta Callao y la línea amarilla; cabe mencionar que existen tramos de vías que se encuentra proyectadas a fin de unir o completar estos eje.

Desequilibrada; su configuración de estructura radial concéntrica, se constituye como un problema puesto que muchas vías se dirigen al centro, que es precisamente donde la demanda de viajes es más intensa. Al llega a la zona del centro de la ciudad que corresponde al centro histórico, las vías se angostan de manera significativa ya que la estructura vial de esta área es antigua y ya consolidada y al no contar con un anillo vial la atraviesan tangencialmente.

Así se configuran dos escenarios uno que corresponde al triangulo de la primera consolidación urbana; que cuenta con una aceptable red vial, de grandes avenidas, relativamente anchas; aunque lo mismo no sucede en el área periférica de generación espontánea, en este los ejes primarios son tangenciales o inexistentes en algunas zonas; existiendo un desequilibrio entre ambas zonas. Esta situación está potenciando las desigualdades sociales en la metrópoli. En palabras de Manuel Herce "el territorio queda privilegiado o marginado por la construcción de infraestructuras, por un lado encontramos redes que son elementos de conectividad y de producción de oportunidades y por el otro lado redes que marginan al espacio por donde pasan".

\subsection{Accesibilidad del destino}

Al ser los desplazamientos consecuencia de la localización de las actividades no basta con estudiar la movilidad, sino que hace falta comprender la accesibilidad, es decir, la facilidad de acceso a estas actividades. En efecto Alfonzo Sanz (1997) mencionaba que si la movilidad es un concepto vinculado a las personas o mercancías que se desplazan; la accesibilidad es un concepto vinculado a los lugares, que además tiene una estrecha relación con las características físicas del espacio y con las posibilidades individuales de los 
ciudadanos para superar las distancias que separan estos lugares. Por tanto "la accesibilidad, además de una dimensión territorial, también es una característica individual en relación con el número de opciones que tienen los diferentes ciudadanos para acceder a los lugares y a las actividades" (Miralles-Guasch, 2002).

\section{Costes ambientales - incremento de la infraestructura de transporte}

Al analizar la relación "motorización - incremento del espacio viario" se verifica que al año 2012 se tenía una oferta de 13,527 km en vías habiéndose incrementado en un 9,6\% en relación al año 2004 según el Observatorio de Movilidad Urbana (OMU) del Banco Interamericano de Desarrollo (BID); este incremento del espacio viario solo supone privilegiar un modos de desplazamiento sobre otro, puesto que el sistema de transporte urbano de Lima solo tiene $34 \mathrm{~km}$ con vías de prioridad de circulación para buses segregados, y a pesar que la población limeña se desplaza mayoritariamente en transporte urbano, eso supone que el resto del espacio viario es compartido entre todos los modos motorizados.

En lo relativo al consumo del espacio, se observa que los vehículos motorizados de transporte individual generan 33,1 millones de vehículos-kilometro, cerca de 5 veces más de los realizados por el transporte urbano. En este sentido, los automóviles y los taxis de uso individual son los vehículos que usan de formas más intensa el sistema vial existente, al representar el $79 \%$ del total de recorridos; este indicador revela que este patrimonio público (sistema vial) es utilizado mayoritariamente por formas individuales de transporte.

\subsection{Condiciones socioeconómicas}

El transporte y la movilidad no solo está relacionado con variables territoriales, también tiene una fuerte relación con las condiciones socioeconómicas como el nivel de renta, el género, la edad, el nivel educativo, el tamaño y la estructura del hogar, las capacidades físicas y mentales, las normas sociales y culturales. Estas variables no actúan de forma aislada, y según cada contexto tienen distinto peso específico como condicionante de la forma de desplazamiento. En las ciudades Latinoamérica el nivel de renta es sin duda una de las principales variables que condicionan la forma de desplazamiento de los ciudadanos.

Lima Metropolitana mayoritariamente este compuesta por el estrado medio bajo con $36,4 \%$, seguido por los estratos medio con $31,1 \%$, medio alto con $14,1 \%$; bajo con $13.1 \%$, y alto con $5,2 \%$ (que constituye el menor nivel). Los estratos alto y medio alto se ubican mayoritariamente en el sector de Lima centro con $21 \%$ y $38 \%$ respectivamente; mientras que los sectores de Lima norte, Lima este, Lima sur y el Callao mayoritariamente están compuestos por los estratos medio, medio bajo y bajo (ver gráfico 5); cabe mencionar que Lima norte nuestra una tendencia de crecimiento poblacional mientras que Lima centro está decreciendo.

\begin{tabular}{|l|l|l|c|l|l|l|l|}
\hline \multicolumn{2}{|c|}{ Estrato } & \multicolumn{2}{c|}{ Personas } & \multicolumn{2}{c|}{ Hogares } & \multicolumn{2}{c|}{ Manzanas } \\
\cline { 3 - 8 } & & Absoluta & $\mathbf{\%}$ & Absoluta & $\mathbf{\%}$ & Absoluta & $\mathbf{\%}$ \\
\hline Alto & A & 381423 & 5.2 & 123165 & 6.1 & 4250 & 4.7 \\
\hline Medio alto & B & 1040030 & 14.1 & 305951 & 15.3 & 8207 & 9.1 \\
\hline Medio & $\mathrm{C}$ & 2298076 & 31.1 & 624359 & 31.2 & 17642 & 19.5 \\
\hline Medio bajo & $\mathrm{D}$ & 2687240 & 36.4 & 685499 & 34.2 & 33460 & 36.9 \\
\hline Bajo & $\mathrm{E}$ & 982231 & 13.3 & 264773 & 13.2 & 27041 & 29.8 \\
\hline Total & & $\mathbf{7 3 8 9 0 0 0}$ & $\mathbf{1 0 0}$ & $\mathbf{2 0 0 3 7 4 7}$ & $\mathbf{1 0 0}$ & $\mathbf{9 0 6 0 0}$ & $\mathbf{1 0 0}$ \\
\hline
\end{tabular}

Grafico 7. Estratificación de ingresos de Lima metropolitana

Fuente: INEI - 2016

\section{Costes sociales - exclusión social}

Los modos de transporte pueden constituirse en medios de exclusión para algunos colectivos de la ciudad así Miralles-Guasch (2009) menciona que "los modelos de movilidad están sujetos a ciertas características sociales, como la adscripción del individuo a un colectivo social, la estructura familiar y el nivel de renta". En Lima metropolitana lo constituyen:

Por nivel de renta: los datos de la encuesta de "Lima Cómo Vamos - 2016" indican que a mayores niveles de renta existe un mayor nivel de motorización, ocurre también que los desplazamientos en medios de transporte urbano o colectivo disminuye y se incrementa el desplazamiento en automóvil propio; mientras que los estratos socioeconómicos de menor renta hacen uso mayoritariamente del transporte urbano colectivo; otro hecho a considerar es que los mototaxis son los medios de transporte principal en el área surburbana, mientras que el taxi es usado principalmente en el centro de la ciudad.

Por género: existe una clara diferencia del acceso a movilidad privada según género, este desplazamiento es mayoritariamente realizado por los hombres con un $78,23 \%$ frente a $21,77 \%$; respecto al desplazamiento en 
bicicleta la relación es $88,06 \%$ frente $11,94 \%$ en el caso de los medios motorizados el acceso preferente que tienen los hombres frente a las mujeres se debe a la estructura social y en el caso de los no motorizados como ir en bicicleta se debe a las malas condiciones o inexistencia de un sistema de carril para bicicletas que permita el acceso de la mayoría. Si comparamos los desplazamiento según estrato socioeconómico podemos ver que a mayor nivel de renta disminuyen los desplazamientos en transporte urbano; lo contrario ocurre con el desplazamiento en automóvil propio este se incrementa. Estos datos muestran que el acceso al automóvil es mayoritariamente un beneficio de los hombres frente a las mujeres, sin embargo las que tienen menor renta son doblemente excluidas. Pero existen colectivos que no están considerados en ninguna encuesta como es el caso de las mujeres que no tienen autonomía económica; este colectivo de mujeres invisibilizadas en las encuestas y cuyas actividades están relacionadas con las actividades domésticas y de proximidad es decir la escala de barrio, con recorridos de 10, 20 y hasta 30 minutos preferentemente caminando.

Por edad: La edad es también otro factor de exclusión del modelo de la movilidad, la relación entre edad y estatus implica un importante aumento de la utilización de automóvil privado a medida que la edad crece la situación profesional juega un papel primordial en los patrones de viaje. Un grupo prácticamente inexistente lo conforman los niños al no existir información específica que analicen los desplazamientos cotidianos, se sabe que los últimos años los niños han ido perdiendo autonomía en sus desplazamientos, quienes son escoltados por sus padres se desplazan principalmente por motivo de estudios. Lima es una ciudad donde la percepción de inseguridad llega a 88,3\% (INEI-2013) lo que indica la percepción generalizada de la calle como un lugar peligroso en parte a la delincuencia urbana pero también al tráfico vehicular.

Por discapacidad: En el Perú viven más de un millón y medio de personas con algún tipo de discapacidad de los cuales 40,75\% se encuentra en Lima Metropolitana (INEI - 2012), que constituyen el 6,8\% de la población total de Lima Metropolitana. Según la primera Encuesta Nacional Especializada sobre Discapacidad del 2012 del INEI, las personas en situación de discapacidad se desplazarse en transporte público 55,5\%; a pie 50,6\%, en taxi 35\%, y moto taxi 19,3\%; cabe indicar que el sistema regular de transporte público no están adaptados para recoger a personas con movilidad reducida. A pesar que en los últimos años se ha implementado el sistema de autobuses denominado "Metropolitano" que cuenta con carril exclusivos y la "línea 1" del metro, estos ofrecen un servicio parcial en cuanto a accesibilidad para personas con movilidad reducida. Si bien durante la implementación de estos, se tomaron en consideración algunos principios de accesibilidad para personas con movilidad reducir al interior de las estaciones, en las calles y espacios públicos entorno a las estaciones así como las vías transversales o conexiones intermodal con otras sistemas de transporte no fueron considerados; un 39,2\% encuentra disculpad de acceso a los medios de transporte público (combis, microbuses, onmibuses); 32\% a las mototaxis; 22\% al Metropolitano; 20,6\% a la línea 1 del metro (según INEI- 2012). Este colectivo presenta los mayores problemas para acceder a las actividades urbanas, puesto que la ciudad no es accesible a sus necesidades de desplazamientos.

\subsection{Características del sistema de transporte}

El sistema de transporte de Lima se compone de transporte privado y transporte público; el primero supone la plena disposición de un medio motorizado para el desplazamiento individual, según la clasificación que realiza la OMU al 2007 dentro de esta categoría están el automóvil, las motocicletas, los taxis de uso privado, y los mototaxis (a nivel de Latinoamérica estos solo existen en Lima y Caracas de manera informal), presentando una de las cifras más altas en cuento al número de taxis 81,826 unidades superando a ciudades como Sao Paulo, Buenos Aires, y Rio de Janeiro; cifra que no registra los taxis informales, así la Municipalidad Metropolitana de Lima ha estimado que podría llegar incluso a 200,000 unidades (entre formales e informales); este exceso de taxis traslada parte de esta flota a la modalidad de taxi colectivo, servicio no reconocido y como consecuencias no autorizados por la Municipalidad Metropolitana de Lima.

Dentro de la oferta de transporte público o colectivo se tiene una variedad que va desde las unidades denominadas taxis colectivas, buses, minibuses, buses segregados, hasta línea del metro (esto dos últimos puestos en operación en los últimos años); si bien el Estado (es el ente responsable de la regular, organizar y gestionar la movilidad en la ciudad), durante casi toda la historia del transporte limeño no ha sido el ente encargado de brindarlo; quienes han brindan el servicio son empresas privadas formales e informales; estos últimos se caracterizan por la invasión de rutas y mayoritariamente presentes en las zonas periféricas.

\section{Costes energéticos - consumo de energía}

A nivel de las ciudades latinoamericanas el área Metropolitana de Lima tiene uno de los más altos consumo de energía por habitante es uno de los más altos, la energía utilizada tiene su origen en los combustibles fósiles y particularmente en el petróleo; el combustible utilizado por el transporte individual mayoritariamente 
es gasolina y en menor cantidad el gas natural de petróleo (GLP) y el gas natural vehicular (GNV) y representa el $64 \%$ mientras frente al transporte colectivo con $36 \%$ que mayoritariamente utiliza diésel y en menor medida gasolina.

\section{Costes ambientales - afecciones al medio ambiente}

\section{Contaminación atmosférica}

La movilidad motorizada es responsable de una gran y creciente proporción de la contaminación del aire en las ciudades y que impactan directamente en la salud de sus ciudadanos. La Organización Mundial de la Salud (OMS) establece que el limite permisibles en las ciudades para partículas finas $\left(\mathrm{PM}_{2,5}\right)$ es de $20 \mathrm{ug} / \mathrm{m}^{3}$ $\mathrm{PM}_{2,5} ; y$, según el reporte del año 2014 el promedio global ha sido de $38,4 \mathrm{ug} / \mathrm{m}^{3}$. Registrándose las concentraciones de contaminación del aire más altas en países en vías desarrollo donde el transporte se ha convertido en una de las principales fuentes de contaminación del aire perjudicial para la salud. Cabe mencionar que Lima Metropolitana llega a $35,7 \mathrm{ug} / \mathrm{m}^{3}$ PM 2.5 muy cerca del promedio global. A nivel de las ciudades de América Latina se encuentra en el puesto tercero de las ciudades más contaminadas después de Tegucigalpa en Honduras y San Salvador en El Salvado con $39,6 \mathrm{ug} / \mathrm{m}^{3} \mathrm{PM}_{2.5}$ con $37 \mathrm{ug} / \mathrm{m}^{3} \mathrm{PM} 2.5$ respectivamente (ver gráfico 6); siendo el promedio regional para América Latina de $14,5 \mathrm{ug} / \mathrm{m}^{3}$.

En cuanto a la Concentración media anual $\left(\mathrm{PM}_{10}\right)$ el máximo permisible según la OMS es de $50 \mathrm{ug} / \mathrm{m}^{3}$ para el caso de Lima Metropolita llega a $88 \mathrm{ug} / \mathrm{m}^{3} \mathrm{PM}_{10}$. Al verificar otros reportes como los del Banco Mundial para el año 2006 indicaba que en el caso del Centro de Lima el PM10 era superior a ciudades de Santiago, Ciudad de México, Sao Paulo y otras; lo que demuestra que la situación no ha cambiado puesto que los índices actuales siguen siendo elevados; cabe recalcar que el Banco Mundial en ese entonces estimo que entre el 70 u 80 por ciento de la contaminación atmosférica de Lima Metropolitana era provocada por los vehículos que circulaban en la ciudad, siendo las principales causas la deficiente calidad de los combustibles, la antigüedad de la flota de vehículos, la falta de control de las emisiones, la sobreoferta de los vehículos entre otros; situación que a la fecha no ha cambiado.

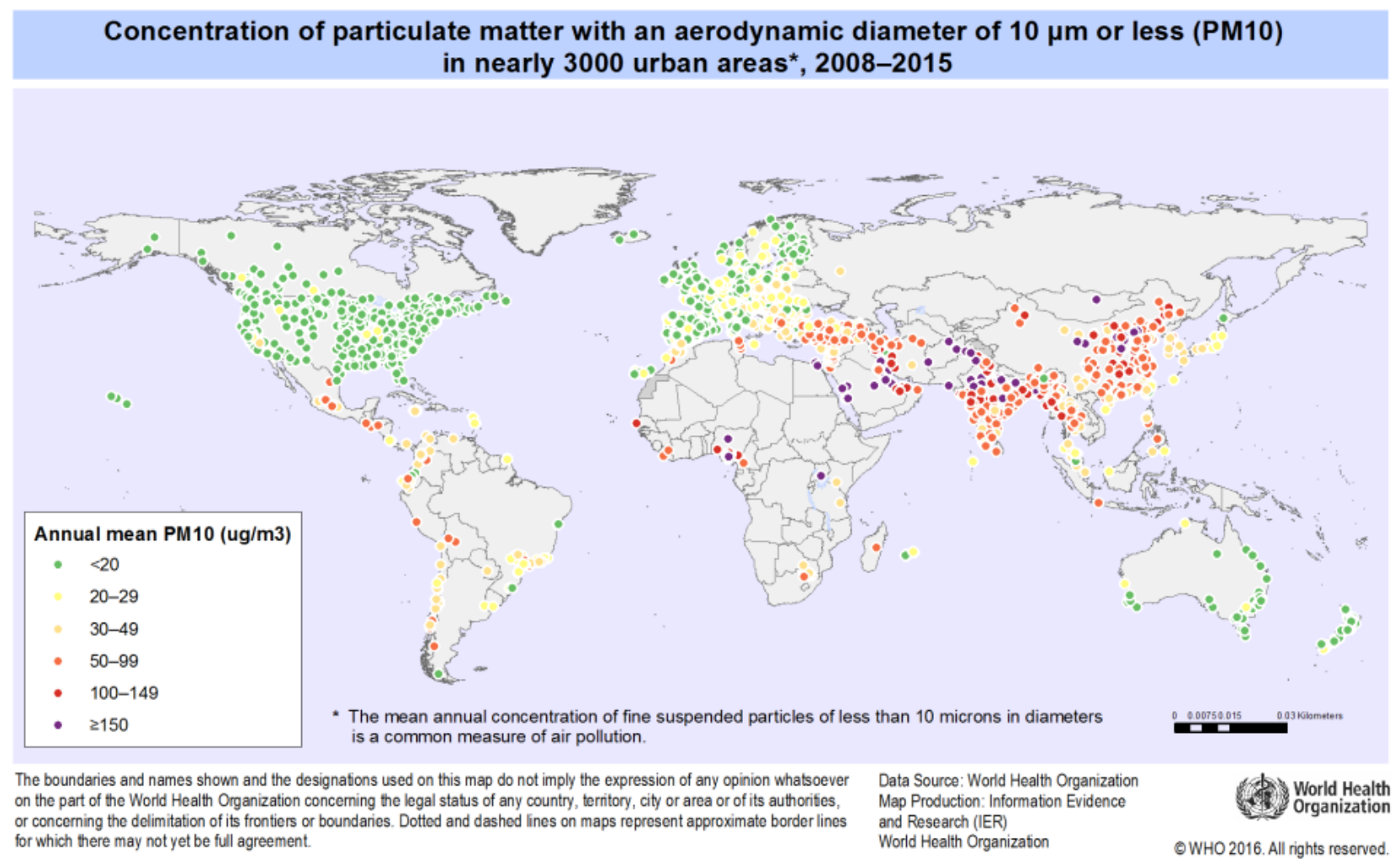

Gráfico 8. Concentración de material particulado (PM10) en áreas urbanas

Fuente: Organización Mundial de la Salud, (2016)

Costes sociales - Inequidad en el acceso a los medios de transporte

Para el contexto de las ciudades latinoamericanas el sistema de transporte público logra movilizar a los sectores más pobres de la población en condiciones de estándares muy bajos. Para el caso específico de 
Lima Metropolitana según Avellaneda (2007) el sistema de transporte colectivo tiene importantes elementos de eficiencia para los sectores populares que facilitan la resolución de sus necesidades de desplazamiento; basándose en el análisis de un sector periférico de la ciudad (barriada de Juan Pablo II³), analiza la eficiencia social del sistema de transporte colectivo llegando a la conclusión que para la población de este sector el sistema de transporte acontece como un importante instrumento de integración y cohesión social; debido fundamentalmente, a tres elementos básicos que caracterizan el sistema de transporte colectivo: su amplia cobertura territorial, su extensa amplitud horaria, y su elevada frecuencia de paso. Por su parte Bielich (2009) cuestiona el término "eficiencia social" usado por Avellaneda; y utiliza el término "eficacia" para calificar el sistema de transporte puesto que a pesar que los usuarios viajan inseguros, incomodos y sin saber cuánto tiempo va a durar el viaje, el sistema de transporte público de Lima logra su principal fin: movilizar a la población.

El área metropolitana de Lima no cuenta con un sistema de transporte integrado, y tal como lo confirman muchos estudios la metrópoli está cubierta en su totalidad por algún modo de transporte público (formal o informal); situación que permite que todos los puntos de la ciudad de alguna formal están articulados, lo que permite corroborar las necesidades de desplazamiento que tienen los ciudadanos y las oportunidades de negocios que esto supone para los operadores;

En este contexto la reciente multiplicación de los viajes como se vio anteriormente solo puede verse como un reflejo de la realización de oportunidades de conexión y es una obligación de equidad democrática que sea posible para todos. Sin embargo a pesar que el sistema de transporte es visto como un problema en la ciudad; los últimos proyectos puestos en marcha y los proyectados a mediano y largo plazo, priorizan solo el sector de Lima centro; el resto se sectores no están cubiertos o solo están de forma parcial; esta situación lleva a la inequidad de acceso al transporte.

\section{LA CAPACIDAD AMBIENTAL EN EL AREA METROPOLITANA DE LIMA}

La capacidad de carga o la cantidad total de transporte que el medio ambiente podría asimilar sin superar un cierto umbral de deterioro estará forzosamente limitada Esteban \& Sanz (1996). Por tanto esta capacidad de carga o capacidad ambiental presentara limites ambientales absolutos, que permitirán determinar si estos han sido o no sobrepasados. Sin embargo, al utilizar terminos como capacidad o límites entramos en un ambito de controversia; puesto que muchas sociedades actualmente conviven, con altos niveles de ruido, intrusión visual, contaminantes atmosferico, o cada vez mayor tiempo de desplazamientos, etc. y probablemente puedan sobrevivir con más afecciones ambientales y sociales a causa de la movilidad motorizada. "El reconocimiento de estos límites implica que la satisfacción de una demanda de transporte indefinidamente creciente - en la cantidad y en la velocidad - no es viable desde el punto de vista ecológico, o simplemente físico" (Sanz, 2010); pero el reconocimiento de estos límites no es el problema sino la acción frente a este, que a la fecha en el área de estudio es ausente.

Al respecto Estevan \& Sanz (1996) mencionan que la única forma de valorar el grado en que se están alcanzando o desbordando los límites ambientales de una determinada actividad, es la de establecer referencias socioculturales; es decir, se trata de examinar cual es la percepción que se tiene del problema en el entorno inmediato de cada colectividad; en términos generales el $51.3 \%$ de la población limeña considera que el transporte público es el según problema más grave de la ciudad (el primero es la inseguridad ciudadana), según Lima Cómo Vamos (2016). Sin embargo, frente a esta insatisfacción no existe por parte de la ciudadanía un rechazo a los problemas del tráfico en la ciudad; esta inacción en parte se debe a lo que Vega Centeno (2016), explica como "el habitante de Lima no se identifica como ciudadano, sino como vecino"; explica que el habitante de Lima, sólo cuando se encuentra entorno a su hogar, experimenta la ciudad como residente donde asume con seguridad la potestad de reclamar ante sus autoridades la gestión de su territorio; pero cuando camina por la ciudad (distinto al entorno de su hogar) es un transeúnte no-residente, donde aprende a diario que la ciudad le es ajena y hostil porque pertenece a otros residentes; experimentando la ciudad no como un ciudadano con derechos y deberes.

\footnotetext{
${ }^{3}$ Juan Pablo II. Formada por cerca de medio centenar de asentamientos situados en el municipio de San Juan de Lurigancho, se encuentra en la periferia de la ciudad. Esta área tiene una población aproximada de 20.000 habitantes la mayor parte de los cuales se encuentra en situación de pobreza o de extrema pobreza. Juan Pablo II es una barriada popular nacida en la década de los años 80 levantada fundamentalmente en base a la autoconstrucción y que todavía hoy sigue en proceso de expansión y consolidación; donde se revela un tipo de movilidad asociada a una forma de transporte perceptiblemente "informal"
} 


\section{CONCLUSIONES}

La configuracion espacial centralizada del AML, de estructura radial concentrica, con una alta especializacion funcional; actua como un iman de actividades, produciendo la necesidad de desplazamientos; esta configuracion es responsable de los mayores costes ambientes y sociales a causa de la movilidad motorizada. Los coste ambientales se asocian con el alto consumo de recursos no renovables como el suelo y energia fosiles; mientras que los coste sociales derivados de la fragmentacion del territorial conducen a la inequidad y exclusion social de amplios estratos de la población.

Los costes ambientales y sociales repercuten en la superacion de los límites ambientales socialmente aceptables en materia de movilidad motorizada en el AML, los que en algunos casos estarian desbordados como es el caso del consumo de recursos no renovable como el suelo y energía fósiles, y los contaminantes atmosféricos. Sin embargo, a pesar de la insatisfacción de la población con el sistema del transporte, al no existir reacción frente a ese reconocimiento de la superación de los ciertos límites, la capacidad ambiental del AML en algunos casos superada y en otros próximo a ser superado. 


\section{BIBLIOGRAFIA}

AGENCIA DE COOPERACIÓN INTERNACIONAL DE JAPÓN - JICA, (2012). "Encuesta de recolección de información básica del transporte urbano en el ara metropolitana de Lima y Callao". República del Perú.

AVELLANEDA, P. (2007). "Movilidad cotidiana, pobreza y exclusión social en la ciudad de Lima". Anales de Geografia (Barcelona), vol. 28 Núm. 2, 9-35.

AVELLANEDA, P. (2009). "La investigación cualitativa en el estudio de las relaciones entre movilidad cotidiana y pobreza en el contexto latinoamericano". Un caso aplicado en la Lima metropolitana. Anales de Geografia (Barcelona) 55, 57-76.

BANCO DE DESARROLLO DE AMÉRICA LATINA (2017). "Crecimiento urbano y acceso a oportunidades: un desafío para América Latina". Corporación Andina de Fomento.

BIELICH, C. (2009). "La guerra del centavo. Una mirada actual al transporte publico en Lima Metropolitana". Lima: Instituto de Estudios Peruanos.

BUCHANAN, C. D. (1973). "Tráfico en las ciudades" [Tráfico en las ciudades]. London (obra original públicada en 1963)

CAMAGNI, R., GIBELLI, M., y RIGAMONTI, P. (2002). "Urban mobility and urban form: The social and environmental costs of different patterns of urban expansion". Ecological Economics, 40(2), 199-216.

CERVERO, R. (1988). "Land-Use Mixing and Suburban Mobility". University of California Transportation Center (Berkeley) 42(3), 429-446.

CERVERO, R. (1991). "Land Uses and Travel at Suburban Activity". University of California Transportation Center (Berkeley), 91.

CERVERO, R., y KOCKELMAN, K. (1997). "Travel demand and the 3Ds: Density, diversity, and design". Elsevier, Vol.2, No 3, 199-219.

CERVERO, R., y RADISCH, C. (1996). "Travel choices in pedestrian versus automobile oriented neighborhoods". Elsevier, Vol. 3, No 3, 127-141.

CHION, M. (2002). "Dimensión metropolitana de la globalización: Lima a fines del siglo XX". Eure, 28(85), 71-87.

ESTEBAN, A.; y SANZ, A. (1996). "Hacia la reconversión ecológica del transporte en España". Madrid: Los libros de la catarata.

FRANK, L. D.; Stone, B., y Bachman, W. (2000). "Linking land use with household vehicle emissions in the central puget sound: Methodological framework and findings". Transportation Research Part D: Transport and Environment, 5(3), 173-196.

FERNANDEZ \& VILELA (2015) "Reflexiones sobre densidad urbana y centralidades en la metrópoli de Lima"

GARBIÑE, H. (2007). "Los costes economicos y sociales de la ciudad de baja densidad. En La ciudad de baja densidad: lógicas, gestión y contención". Barcelona: Diputació Provincial de Barcelona, 203-241.

GONZALES, E., y DEL POZO, J. M. (2012). "Lima, una ciudad policéntrica. Un análisis a partir de la localización del empleo". Investigaciones Regionales (Lima), 23, 29-52.

HERCE, M. (2009). "Sobre la movilidad en la ciudad :propuestas para recuperar un derecho ciudadano". Barcelona: Editorial Reverté.

HERCE, M., y MAGRINYÀ, F. (2013). "El espacio de la movilidad urbana". Buenos Aires: Café en las ciudades.

MATOS MAR, J. (2012). "Perú: Estado desbordado y sociedad nacional emergente". Lima: Centro de investigacion, Universidad Ricardo Palma.

MIRALLES-GUASCH, C. (2002). "Ciudad y transporte. El binomio imperfecto". Barcelona: Ariel.

POLZIN, S. (2004). "The Relationship Between Land Use, Urban Form and Vehicle Miles of Travel: the State of Knowledge and Implications for Transportation Planning". University of South Florida, 1-28.

POUYANNE, G. (2004). "The motives of mobility : an infra-urban level analysis. The case of Bordeaux, France". Université Montesquieu Bordeaux IV, 9, 1-29.

SANZ, A. (1997). "Movilidad y accesibilidad: un escollo para la sostenibilidad urbana". Madrid.

SANZ, A. (2010). "Transporte, economía, ecología y poder. La economia del transporte desde un enfoque eco integrador". Grupo de Estudios y alternativas 21, S. L. Ekonomiaz N1 73, 1er cuatrimestre.

SANZ, A., VEGA, P., y MATEOS, M. (2014). "Cuentas ecológicas del transporte". Libros en Acción (la editorial de Ecologistas en Acción.

VEGA-CENTENO, P. (2017). "La dimensión urbana de las centralidades de Lima Norte: Cambios y permanencias en la estructura metropolitana". Eure, 43(129), 5-25. 